Mens

revue d'histoire intellectuelle de l'Amérique française

\title{
Antoine Boisclair. L'École du regard : poésie et peinture chez Saint-Denys Garneau, Roland Giguère et Robert Melançon, Montréal, Fides, 2009, 426 p.
}

\section{France St-Jean}

Volume 11, numéro 2, printemps 2011

URI : https://id.erudit.org/iderudit/1023381ar

DOI : https://doi.org/10.7202/1023381ar

Aller au sommaire du numéro

Éditeur(s)

Centre de recherche en civilisation canadienne-française

ISSN

1492-8647 (imprimé)

1927-9299 (numérique)

Découvrir la revue

Citer ce compte rendu

St-Jean, F. (2011). Compte rendu de [Antoine Boisclair. L'École du regard : poésie et peinture chez Saint-Denys Garneau, Roland Giguère et Robert Melançon,

Montréal, Fides, 2009, 426 p.] Mens, 11(2), 133-136.

https://doi.org/10.7202/1023381ar 


\section{Antoine Boisclair. L'École du regard: poésie et peinture chez Saint-Denys Garneau, Roland Giguère et Robert Melançon, Montréal, Fides, 2009, 426 p.}

«Débarrasser la vision des mots, des idées, de la pensée; retrouver le regard du premier matin du monde qui permettrait de simplement voir ce qui est. " Ces mots, les premiers d'Antoine Boisclair, donnent le ton à cet imposant ouvrage résultant de sa thèse de doctorat. Cette ambition, voire ce leitmotiv que partagent les écrivains $\mathrm{du} \mathrm{xx}^{\mathrm{e}}$ siècle, particulièrement ceux qui espèrent retisser le lien (rompu) entre le visible et le dicible pour pouvoir " mieux voir par la parole" (p. 12), Boisclair se l'approprie afin de rendre compte "des inflexions modernes de l'ut pictura poesis" (p. 37). À travers la lecture, principalement, mais non exclusivement, des dialogues poétiques et théoriques qu'entretiennent les poètes Hector de Saint-Denys Garneau, Roland Giguère et Robert Melançon avec la peinture, l'auteur réussit à brosser un portrait détaillé des processus de vision (ou modes de visibilité) qui ont marqué leur époque respective. En allant au-delà du lieu commun postulant que « les peintres ont tracé la voie aux écrivains » (p. 18), Boisclair analyse l'articulation des liens entre le voir et le dire que proposent certains poètes québécois et qui ont contribué au développement de nouvelles esthétiques.

L'ouvrage est divisé en trois parties, trois parties correspondant aux trois régimes de visibilité : " apprendre à voir ", "donner à voir " et " faire voir " que constitue cette " école du regard " et qui sont exemplaires, pour les années 1920-1930, du renouveau du paysage canadien; pour les années 1948-1960, des nouvelles esthétiques nées autour et à la suite du Refus global ainsi que des années 1980-2000 durant lesquelles cohabitent plusieurs esthétiques dont le seul point commun est le rejet de la tradition de rupture qui sous-tend toute la période moderniste.

Dans la première partie, consacrée à Saint-Denys Garneau, l'auteur nous présente l'" apprendre à voir " par un bref aperçu sur le dialogue qu'entretient la poésie canadienne-française avec la 
peinture, depuis Tableau de la Cataracte de Niagara de Joseph Mermet, paru dans le Spectateur en 1815, jusqu'à Fleur de gel de René Chopin, publié en 1913. Toutefois, et Boisclair en fait la démonstration, quoique les poètes évoluant sur le territoire québécois aient toujours eu un intérêt pour la peinture et l'acte de peindre, la réflexion qu'ils ont menée n'a laissé à la postérité rien de « substantiel ». C'est donc avec le questionnement de Saint-Denys Garneau que s'amorce réellement dans le Québec francophone le dialogue entre le dire et le voir, ou, plus justement dans le cas de Saint-Denys Garneau, entre le voir et le dire, puisque, comme en témoignent les textes invoqués par Boisclair, l'éducation du regard chez l'auteur de Regards et jeux dans l'espace passe d'abord par l'interrogation sur l'acte de peindre et sa matérialité. En cela, le poète s'est largement nourri autant des œuvres de peintres tels Maurice Cullun, Edwin Holgate et Louis Muhlstock, que de celles de Paul Cézanne et d'Henri Matisse, que des discours sur l'art du philosophe Jacques Maritain, de l'historien de l'art Élie Faure et, plus près de nous, du critique d'art Henri Girard. Ce qui explique sans doute que, malgré sa volonté de renouvellement dans la manière de voir, faisant de lui un moderne, Garneau reste attaché à la représentation d'un sujet concret. Il n'en demeure pas moins, cependant, que son désir d'" éduquer " son regard (et celui de l'élite) aura ouvert la voie à l' " apprentissage " du voir, ce qui nous amène à la deuxième période de l'" École du regard ".

Poursuivant son analyse du rôle du regard dans la conception de nouvelles esthétiques, Boisclair met en évidence le fait que le régime de visibilité que développe Roland Giguère, sans rompre totalement avec son prédécesseur, vise non plus l'apprentissage du regard par l'intermédiaire de l'espace de la nature, mais plutôt par l'exploration des « territoires intérieurs de l'imaginaire » (p. 46). Cette place importante accordée à l'expression de l'inconscient permet donc de "donner à voir " la part de l'invisibilité sur laquelle le regard ne s'est pas encore posé (p. 203), part de l'invisibilité que le phénoménologue Jean-Luc Marion a conceptualisée sous le terme de l'« invu ». De même, Boisclair le souligne, l'introspection de l'être 
qui marque la production tant poétique que picturale de Giguère aura permis de briser " la censure exercée par la conscience sur le visible " (p. 47), qui était encore présente dans la manière de voir de Saint-Denys Garneau. Dans cette deuxième partie consacrée au " donner à voir ", l'auteur de L'École du regard met aussi en lumière l'apport des poètes Alain Grandbois, Gilles Hénault, Claude Gauvreau et Éloi de Grandmont, de même que celui de l'historien d'art Maurice Gagnon, qui ont précédé Giguère dans la formulation de cette manière de regarder. Il y est aussi question de la dette de l'artiste envers le surréalisme, que ce soit à travers l'œuvre de Paul Éluard ou celles d'Alfred Pellan et d'Albert Dumouchel. Toutefois, et l'on voit là toute la finesse de l'étude, Boisclair rappelle que le dialogue entre le dicible et le visible par un "illumineur " tel que Giguère, et qui marque un tournant dans la compréhension identitaire du sujet à travers le regard, est largement décrié par certains poètes, telle Fernande Saint-Martin, de même que par les Plasticiens et les Néo-Plasticiens pour qui le seul voir possible se trouve dans le matériau utilisé, les formes et les structures de l'œuvre.

Cette étude des inflexions modernes qu'ont données les poètes québécois à l'ut pictura poesis se conclut avec le parcours de Robert Melançon. Dans cette troisième partie, il est largement question du " faire voir ", celui de l'essence même des choses ou, pour employer les mots de Boisclair, de " ce qui est ». Toujours selon Boisclair, le projet esthétique que précise Melançon s'inscrit à la suite du regain d'intérêt que suscite la phénoménologie, à propos notamment de l'apparition et de l'essence des choses. Il emprunte aussi la voie tracée par les poètes Robert Marteau, Fernand Ouellette et Jacques Brault pour qui il importait « de renouer avec le sens et la présence » (p. 313) du sujet. On ne s'étonne donc pas de constater que l'auteur de Peinture aveugle et de Le dessinateur se soit intéressé à l'immédiateté et à la quotidienneté des choses que l'œil, qui avait pourtant été habitué à sonder les profondeurs de l'être, ne voit plus. Se distanciant de l'esprit de révolte qui accompagne l'œuvre de Giguère et, dans une moindre mesure, celle de Saint-Denys Garneau, le régime de visibilité de 
Melançon s'ancre dans la réalité concrète et affiche un certain plaisir de vivre. En fait, comme l'affirme Boisclair, la manière de voir de Melançon "vise moins à "réinventer le monde" qu'à mieux l'habiter " (p. 390).

En se proposant d'examiner de quelle façon la peinture fait œuvre d' " école du regard " chez les poètes québécois du Xxe siècle, Antoine Boisclair offre ici plus qu'" une étude d'envergure sur l'histoire de la relation entre la poésie québécoise et la peinture " (p. 19). Il fait la démonstration que le dialogue entre le dire et le voir que tiennent les poètes québécois rend compte du caractère distinct de l'esthétique québécoise, et cela, tout en s'inscrivant dans le parcours de l'ut pictura poesis, depuis Horace, qui conçoit « la poésie [...] comme une peinture ", jusqu'à ses inflexions modernes suscitées à la suite de la critique systématique développée par Lessing dans son Laocoon. Convoquant autant les théories sémiologiques, les études sur la perception que la phénoménologie, Boisclair fait ressortir un à un les enjeux littéraires et philosophiques qui témoignent de la volonté des Garneau, Giguère et Melançon de " mieux voir par la parole " (p. 12).

Ce qui distingue L'École du regard de tant d'autres ouvrages abordant la question du rapport entre l'image et le texte est sans doute le fait que l'auteur ne s'est pas contenté de mettre au jour les points de rupture; il a également tenu compte de la prégnance des références picturales qui ont ponctué l'énonciation du discours des poètes québécois, depuis l'époque de La Relève (1934) jusqu'au Paradis des apparences (2004). Bref, il s'agit là d'une étude remarquable, d'un « regard " qui, quoique découlant d'une perspective littéraire, nous «donne à voir » un Québec moderne qui, tour à tour, a pris conscience du territoire occupé, de la profondeur de son être et de sa relation avec le monde. À lire et à relire pour en découvrir toute la subtilité. 